Itinéraires Itinéraires

Littérature, textes, cultures

2012-1 | 2012

Genres et avant-gardes

\title{
Claude Cahun : la subversion des genres comme arme politique
}

Alexandra Bourse

\section{(2) OpenEdition}

Journals

Édition électronique

URL : http://journals.openedition.org/itineraires/1300

DOI : $10.4000 /$ itineraires. 1300

ISSN : 2427-920X

Éditeur

Pléiade

\section{Édition imprimée}

Date de publication : 1 septembre 2012

Pagination : 137-145

ISBN : 978-2-296-55776-5

ISSN : $2100-1340$

Référence électronique

Alexandra Bourse, "Claude Cahun : la subversion des genres comme arme politique ", Itinéraires [En ligne], 2012-1 | 2012, mis en ligne le 01 septembre 2012, consulté le 19 avril 2019. URL : http:// journals.openedition.org/itineraires/1300; DOI : 10.4000/itineraires.1300

\section{(ब) $(\Theta \Theta$}

Itinéraires est mis à disposition selon les termes de la licence Creative Commons Attribution - Pas d'Utilisation Commerciale - Pas de Modification 4.0 International. 


\title{
Claude Cahun : la subversion des genres comme arme politique
}

\begin{abstract}
This article is about gender subversion and political rebellion in the artistic works of Claude Cahun. Her most achieved book is entitled Aveux non avenus. It resists all literary categorisation and gives an impression of disorder. Actually, this is how Claude Cahun suggests that our mind, our sexuality, our identity can not be explained in terms of established classifications. This rebellion against social conventions also appears in political texts that Claude Cahun wrote in collaboration with the Surrealist group. In an important pamphlet, Les Paris sont ouverts, Cahun shows the political implications of gender subversion. Furthermore, she concretely applied this strategy during the Second World War and the German Occupation in Jersey with the help of her lover, Suzanne Malherbe. Adopting the surname of "The Unnamed Soldier" on pamphlets she wrote, Cahun used masks to fight for freedom.
\end{abstract}

Keywords : genre/gender subversion, lesbianism, disguise, surrealist political fights, feminine resistance

Mots clés : subversion des genres (littéraires et sexuels), artiste lesbienne, travestissement, luttes politiques des surréalistes, femmes dans la Résistance

En 1993, le musée de Jersey a rendu hommage au couple formé par Claude Cahun et Marcel Moore dans une exposition intitulée « Sœurs surréalistes : une histoire extraordinaire d'art et de politique ». Il s'agissait de mettre en évidence un événement peu commun : l'accès à la création et à la lutte politique de deux femmes lesbiennes et leur reconnaissance par le groupe surréaliste. Nous parlerons surtout de Claude Cahun, mais justice serait à rendre à Suzanne Malherbe car les deux femmes ont en réalité presque toujours créé et lutté ensemble. Choisir pour pseudonyme « Claude Cahun » (à la place de Lucy Schwob) n'est pas anodin : il s'agit, tant dans les œuvres littéraires que dans le combat politique, de revêtir un masque identitaire, capable de renier l'attachement à une catégorie donnée, qu'elle soit de genre ou de classe. La pratique de l'autoportrait 
photographique est sans doute ce qui retient actuellement le plus l'attention des exégètes au sujet de Cahun. Néanmoins, cette pratique n'a de sens que dans un dialogue prolongé avec un travail scripturaire dont 1'exigence ultime est de subvertir la notion même d'identité. Cette remise en cause de l'identité est tout de même à nuancer. Dans le contexte de la montée des fascismes, puis sous l'Occupation, Cahun défend les valeurs démocratiques françaises, mais toujours en utilisant son arme favorite : le travestissement. Cahun prête une attention toute particulière à ce qui a lieu autour d'elle. Elle est sensible à la situation socio-politique dans le contexte international de son époque : les conséquences de la crise de 1929, l'exacerbation des antagonismes de classes, les mouvements de masse, le renforcement du Parti communiste. Sa rencontre avec les surréalistes et notamment son amitié avec André Breton la conduisent à écrire et à signer des pamphlets politiques dirigés contre l'installation du fascisme allemand mais aussi contre le durcissement stalinien du Parti communiste.

Il s'agira de montrer comment Claude Cahun, évoluant dans le sillage du groupe surréaliste tout en conservant son indépendance, a proposé de multiples pratiques artistiques subversives faisant de la mise à mal des normes de genre une arme politique. Deux ouvrages majeurs en témoignent plus particulièrement : il s'agit d'Aveux non avenus (1930) et des Paris sont ouverts (1934) qui, situés de part et d'autre de la rencontre avec le groupe surréaliste, mêlent étroitement analyse de soi, travail sur le corps et lutte politico-sociale et proposent même un vaste réquisitoire contre une vision monolithique du sujet.

L'œuvre la plus aboutie de Claude Cahun, à savoir Aveux non avenus, est une mise en œuvre avant l'heure de ce qu'elle définira plus tard comme « poésie d'action indirecte ». En effet, il s'agit d'en passer par l'écriture pour lutter contre différents systèmes d'oppression : le texte dénonce déjà l'antisémitisme, l'entreprise de catégorisation des individus en fonction de leur genre, la dévalorisation des femmes. L'auteur marque une attention toute particulière à l'écriture, pratique artistique qu'elle valorise le plus, estimant que, contrairement aux autres formes d'art, elle engage tout entier celui qui la pratique, jusqu'au sacrifice de lui-même. Don absolu de soi, l'écriture demande un investissement total; on entre dans l'ordre de l'écriture et on n'en sort plus. La photographie s'allie à cette entreprise pour dénoncer les illusions du monde et en jouer sur un mode ironique et humoristique. Il s'agit, par l'entremise de deux médias, d'exercer l'œil, mais aussi de s'imposer une discipline corporelle contraignante afin de ne donner toute la place qu'aux aventures de l'esprit. Il faut effacer le corps pour augmenter l'acuité intellectuelle et spirituelle : « Je ne voudrais coudre, piquer, tuer, qu'avec l'extrême pointe. Le reste du corps, la suite, quelle 
perte de temps! Ne voyager qu'à la proue de moi-même ${ }^{1}$. » L'exigence intellectuelle de Cahun est extrêmement poussée, jusqu'à une véritable ascèse. Ce détachement de la corporéité lui permet dans le même temps de se servir du corps comme d'un terrain d'expérimentation. Le recueil Aveux non avenus se présente à la manière des Essais de Montaigne, propose une expérience personnelle qui pourrait servir à un groupe beaucoup plus large. L'un des objectifs de cette expérience vise à se départir des genres (au sens de gender) et des contraintes sociales qu'ils imposent en écartant entre autres les normes de genres attachés aux genres littéraires. Aveux non avenus échappe ainsi à la catégorisation : on y trouve des récits de rêves, des saynettes théâtrales, ce qui ressemble à des morceaux d'essais philosophiques, des poèmes. La forme de l'œuvre elle-même reflète un désengagement face à tout cadre normatif. En même temps, ces changements de tons et de formes signalent la lutte constante d'un esprit tourmenté cherchant la meilleure manière de dire et de dénoncer.

Claude Cahun revient sur cette expérimentation dans Les Paris sont ouverts en expliquant, en note, l'utilité d'une expérience individuelle de la poésie dans la lutte collective :

Je rêve ou j'imagine ailleurs des corps, parmi lesquels parfois mon propre corps. Ces objets rêvés ou imaginés se trouvent toujours situés dans l'espace par rapport à mon corps vivant qui me sert en quelque sorte de boussole. Cette situation des objets dans l'espace est ce que je retiens le plus fortement, tous autres détails de mes rêves ou images, tous autres souvenirs effacés. Pourtant le rapport entre ces images et moi existe effectivement dans le temps et non dans l'espace. Comment rattacher à un mécanisme de classe ce mythe, cette particularité qui ne m'est pas particulière sans doute, que je puis fort bien avoir en commun, subir en commun avec des ouvriers, même conscients? Mes adversaires nieront qu'il y ait le moindre intérêt à éclaircir la chose, à la réduire. Pourtant on n'est pas même en droit d'affirmer qu'une entreprise aussi futile pour les uns, aussi folle pour les autres, aussi ridicule pour les uns et les autres, ne mènerait à rien qui présente le moindre intérêt ${ }^{2}$.

Libérer le corps des catégories est déjà un acte politique. Se mettre complètement à nu, c'est tenter par la même occasion de remédier à un paradoxe évident auquel se confrontent les surréalistes au moment de l'affaire Aragon : l'écrivain révolutionnaire est fatalement conduit à écrire pour ou contre la classe qu'il combat (car on écrit pour ceux qui savent lire et qui en ont le loisir). Du même coup, il faut sans cesse faire l'effort d'écrire contre soi-même ${ }^{3}$. L'écriture de Cahun est en quête constante

1. Claude Cahun, Aveux non avenus [1930], repris dans Écrits, édition présentée et établie par François Leperlier, Paris, Jean-Michel Place, 2002, p. 178.

2. Claude Cahun, Les Paris sont ouverts [1934], ibid., p. 527.

3. Claude Cahun, article publié dans la revue Commune, $\mathrm{n}^{\circ}$ 4, décembre 1933, en réponse à la question « Pour qui écrivez-vous? » : « C'est assez dire que j'écris, que je souhaite écrire avant tout contre moi » (ibid., p. 538). 
de l'intranquillité, de la situation précaire qui lui permet d'être en état permanent de vigilance contre les habitudes de l'esprit, le confort. Cahun remarquait déjà en 1930 dans Aveux non avenus que la société bourgeoise, capitaliste, s'endormait dans sa foi en un dieu Argent. Cet amour de l'avoir menace l'art et c'est bien aux artistes justement de rester attentifs. Elle exigeait avec férocité que les bourgeois « envoient leurs âmes au diable! Non pas leurs âmes, mais ce qui leur en sert, non ces sœurs solennelles, mais cette conscience quotidienne : esprit, bonheur, stabilité, amour bien fondé, souvenirs, habitudes, avenir, devenir », et elle soutenait en postscriptum qu' ' à présent, [elle] exist[ait] autrement $»^{4}$.

Signe de cet inconfort recherché, les formulations de Claude Cahun restent toujours volontairement sujettes à caution. Aucune véritable assertion ne peut y être décelée. Ainsi, lorsqu'elle se place du côté des révoltés dans Aveux non avenus laisse-t-elle tout de même place à une forme de relativisme :

\footnotetext{
Chacun veut que sa recette soit l'unique espoir de salut. Les joyeux garçons n'admettent gentiment que légèreté, désinvolture. Les affligés, les révoltés (ne suis-je pas des vôtres?) - nous exigeons le rêve, griffes dehors ou patte de velours. Un philosophe dresse la liste de ses qualités négatives et positives. Ce seront les péchés nouveaux, les valeurs universelles. Enfin pour les sceptiques, pour les croyants (que ce soit en Dieu ou en diables), il n'est tel que de douter, que de croire ${ }^{5}$.
}

Cahun a beau se placer du côté des révoltés, elle n'estime pas détenir la vérité absolue et met donc sur le même plan différents types de postures intellectuelles sans trancher entre les unes et les autres. Selon elle, ce qu'il faut absolument préserver c'est la liberté d'action par rapport à tout mouvement. Il s'agit toujours de circuler « librement dans l'espace intermédiaire ${ }^{6} \gg$ et de résister jusqu'à mettre sa vie en danger.

Un lien direct est établi par Claude Cahun entre une œuvre de fiction, Aveux non avenus, et un texte engagé du point de vue politique, Les Paris sont ouverts. Le désordre apparent d'une œuvre comme Aveux non avenus, inclassable du point de vue du genre littéraire, manifeste la volonté de conserver une liberté de ton, même si Cahun affiche une préférence pour le communisme trotskiste. Il s'agira dès lors de montrer que Les Paris sont ouverts n'est pas seulement un pamphlet politique, mais s'apparente également à un projet poétique, qui fait le point sur les tentatives artistiques de Claude Cahun jusqu'en 1934 et asseoit une forme d'art poétique.

Suite à de violentes attaques contre la plupart des intellectuels de gauche et d'extrême-gauche, dont les surréalistes, contre tous ceux qui ne

4. Claude Cahun, Aveux non avenus, op. cit., p. 191.

5. Ibid., p. 376.

6. Ibid., p. 377. 
se soumettaient pas aux directives de l'Union internationale des écrivains révolutionnaires (UIER), le Parti communiste délègue en janvier Paul Vaillant-Couturier, Léon Moussinac, Paul Nizan et Jean Fréville pour animer une "Association des écrivains et artistes révolutionnaires » (AEAR). L'artiste se trouve sommé de donner un sens à son art dans un contexte politique tourmenté. La question majeure est de savoir quel impact peut avoir l'écriture, et plus particulièrement la pratique poétique, dans un contexte de révolte sociale. Que peut la poésie dans le cadre d'une lutte des classes? N'est-ce pas se désengager et ne vivre qu'en individualiste que de faire de la poésie? C'est à ce faisceau de questionnements que se trouvent confrontés les membres du groupe accusés d'être contre-révolutionnaires, notamment par Aragon faisant paraître un manifeste dans L'Humanité le 22 mars 1932 intitulé « La littérature et l'art prolétarien révolutionnaires doivent être un puissant moyen de propagande et d'éducation révolutionnaires des masses ».

Claude Cahun s'investit aux côtés des surréalistes dans les années 1930, avec le souci de s'engager auprès d'eux au moment où le groupe se trouve menacé d'implosion et ce, après le formidable retentissement qu'a eu le départ d'Aragon. Son pamphlet, Les Paris sont ouverts, est une réponse envoyée aux incartades d'Aragon qui avait rompu avec le surréalisme à la suite du Congrès international des écrivains révolutionnaires de Kharkov organisé en novembre 1930. Lorsqu'en 1934 Claude Cahun, alors membre de l'AEAR, rédige Les Paris sont ouverts, elle signe en conservant son pseudonyme épicène de « Claude». Ce geste montre bien l'intrication entre revendications politiques déclarées (soutien du prolétariat, revendications trotskistes) et voilées (la mise en question des genres, la possibilité pour un être entre deux genres de faire entendre sa voix). Signalons tout de même que si ce pamphlet politique de Cahun trouve des lecteurs attentifs, c'est aussi parce que le pseudonyme choisi par l'artiste la fait passer pour un homme. Même chose lorsque Breton signale la participation de Cahun à "Contre-Attaque », union de lutte des intellectuels révolutionnaires, groupe constitué en 1935. C'est dans les deux cas la subversion identitaire qui lui permet de prendre part aux mouvements révolutionnaires.

Par ailleurs, Claude Cahun met ses pratiques plastiques au service de ses revendications. Dans Poupée (1936), série de photographies d'objets, elle se moque du Parti communiste français en le traitant de marionnette mercenaire de Staline. Ces clichés sont produits en septembre 1936, après que Paris a eu écho des procès de Moscou et que le Parti communiste français a décidé de continuer à soutenir l'Union soviétique stalinienne. Les marionnettes aux allures de soldats grotesques sont formées à partir de gros titres de l'Humanité.

La participation à l'AEAR et la rédaction d'un texte sur le rôle de la littérature en contexte révolutionnaire qui trouvera son aboutissement dans Les Paris sont ouverts permettent à Claude Cahun de conjuguer 
préoccupations politiques et littéraires. Elles lui permettent également de revenir sur le rôle qu'elle confère à la photographie. Dans ce cadre, Les Paris sont ouverts se présente à la fois comme un bilan et comme la confirmation de la tentative exploratoire d'Aveux non avenus.

La participation aux luttes politiques exige également de Claude Cahun qu'elle réfléchisse au rôle qu'elle attribue à la pratique artistique et notamment à la poésie. Il s'agit pour elle de donner un sens à une pratique qui pourrait être considérée comme inutile face aux tâches réclamées par la propagande marxiste. Or, la question de l'utilité de la poésie n'est pas valable pour Cahun qui estime qu' ' ayant existé historiquement en tous temps et lieux [elle] semble indéniablement un besoin inhérent à la nature humaine, voire animale, besoin lié sans doute à l'instinct sexuel ${ }^{7} \gg$. Aux yeux de l'artiste, la poésie transcende la contingence sociale et politique, s'impose au plus profond de l'humain, au plus animal. On comprend du même coup que l'analyse de soi puisse être un matériau fécond à une métamorphose du poétique et du politique.

L'évolution poétique et l'évolution sociale se suivent, se subordonnent l'une à l'autre. En rend compte, non pas le «contenu manifeste de la poésie », mais son " contenu latent», estime Cahun, reprenant la terminologie utilisée par Tristan Tzara. La force révolutionnaire des poètes se trouve dans le contenu latent de leurs œuvres. En revanche, leur contenu manifeste ne peut être que provisoire, précaire et est réservé aux journalistes ou aux orateurs car, de leur côté, « les poètes agissent à leur façon sur la sensibilité des hommes. Leurs atteintes sont plus sournoises; mais leurs coups les plus détournés sont parfois mortels ${ }^{8} \gg$. Claude Cahun s'en prend à Aragon qui estime quant à lui que c'est la technique qui décide de tout. Cette idée est dangereuse car « la critique mettant l'accent sur le contenu idéologique manifeste des poèmes est favorable aux tricheurs, à tous ceux qui veulent passer pour autres qu'ils ne sont, et qui pour cela se livrent à une sorte de surenchère idéologique ${ }^{9} »$. Ce passage par l'idée de contenu latent est central pour Cahun, puisqu'il lui permet ici de relier ses deux pratiques artistiques privilégiées. En effet, poésie comme photographie demandent à être révélées pour délivrer le combat secret qu'elles mènent. Le choix des photomontages et de l'énigme qu'ils dessinent va dans ce sens, celui d'un passage nécessaire par l'imaginaire pour comprendre que ce qui est montré appelle le déchiffrement.

Il serait utile de faire [...] l'analyse de poèmes activité d'esprit. Leur traduction produirait parfois, j'en suis persuadée, des révélations de ce genre : un homme a cru photographier les cheveux mêlés de brins de paille de la

7. Claude Cahun, Les Paris sont ouverts, op. cit., p. 507.

8. Ibid., p. 508. Voir Tristan Tzara, «Essai sur la situation de la poésie », Le Surréalisme au service de la révolution, $\mathrm{n}^{\circ}$ 4, décembre 1931.

9. Claude Cahun, Les Paris sont ouverts, op. cit., p. 509. 
femme qu'il aime, endormie dans un champ. Le cliché révélé, apparaissent mille bras divergents, des poings brillants, des armes; on s'aperçoit qu'il s'agit d'une émeute ${ }^{10}$.

La poésie garde son secret, ou plutôt, elle secrète une révolution. Dans ce cadre, il s'agit de privilégier une action indirecte prônant une poésie participative : provoquer le désir du lecteur, celui d'aller plus loin, de prolonger le poème en action physique ou de l'esprit.

La théorisation et la poétisation de l'indéfinition, qui devient le mot d'ordre de Claude Cahun dès Aveux non avenus et trouve un prolongement idéologique dans Les Paris sont ouverts (pamphlet dans lequel apparaît significativement en sous-titre « La poésie garde son secret »), ont été utilisées concrètement comme arme de résistance sous l'Occupation. À Jersey, Cahun a établi une perméabilité entre un principe esthétique, éthique et une pratique ancrée dans le réel.

C'est pendant l'Occupation que Claude Cahun passe de la théorisation à l'action et qu'elle a recours au travestissement, déjà utilisé dans ses photomontages et ses œuvres scripturales. Dans un contexte de surveillance contraignante, Cahun refuse de dire son nom, celui de Schwob, car elle ne reconnaît pas de légitimité aux noms propres qui ne rendent pas justice à l'humain. Il s'agit au contraire de le rendre à sa complexité en reconnaissant sa multiplicité : « Les signes ont-ils un sexe? Mon multiple est humain. Un signe hermaphrodite ne suffirait pas à le rendre (à lui rendre justice) $)^{11} »$. Penser l'individu dans sa multiplicité, c'est du même coup rejeter toute assignation identitaire. Dans ce cadre, et malgré le danger, Cahun use du travestissement et du pseudonyme dans la résistance contre l'occupation allemande. Évidemment, cette résistance va de pair avec une révolte contre l'antisémitisme qu'elle dénonçait déjà dans Aveux non avenus avec une ironie mordante. Il ne faut pas oublier que le premier pseudonyme de Cahun a été « Courlis », nom d'un oiseau au bec très long, faisant référence au stéréotype antisémite du Juif au nez crochu. Cahun avait souvent joué de ce racisme pour le détourner et en faire le participe d'une spiritualité à contre-courant : «Moi, juive au point d'utiliser mes péchés à mon salut, de mettre en œuvre mes sous-produits, de me surprendre continuellement, l'œil en crochet, au bord de ma propre poubelle ${ }^{12}$ ! "L'artiste a laissé un texte évoquant la période de la Résistance ainsi que les affres de la prison (Cahun et Moore avaient été condamnées à mort pour leurs actes de résistance et n'avaient échappé à l'exécution que grâce à l'intervention d'un ami, Gaston Ferdière, auquel le texte est dédié). Le Muet dans la mêlée (mars 1946) et Confidences au miroir (inédit 1945-1946) retracent les événements dont la réalité est subvertie par le récit des angoisses et des cauchemars de

10. Ibid., p. 510.

11. Claude Cahun, « Confidences au miroir» [1945-1946], dans Écrits, op. cit., p. 586.

12. Claude Cahun, Aveux non avenus, op. cit., p. 211. 
Cahun de mars 1946. Dans Le Muet dans la mêlée, Claude Cahun brosse le portrait des habitants de Jersey à l'époque de l'Occupation, surtout celui des femmes dont elle relève une certaine masculinisation, celles-ci portant une « veste semi-masculine » ou comptant parmi elles « des paysannes et aussi des filles et des femmes de la bourgeoisie à caractère gentleman farmer $\gg{ }^{13}$. Jersey devient ce microcosme dans lequel la neutralisation des genres a lieu en plein contexte de résistance. À cette neutralisation répond une conquête de l'anonymat, Cahun et Moore ayant baptisé leur maison « La ferme sans nom ». De la même manière, lorsqu'elles écrivent des tracts hostiles au nazisme, les deux femmes se font passer pour « le Soldat sans nom ». L'indéfinition est l'arme de la résistance, elle permet tous les mouvements et toutes les métamorphoses. Une anecdote est à ce titre extrêmement intéressante. C'est lorsque Claude Cahun use de son nom véritable, c'est-àdire celui que la société lui a conféré, « Lucy Schwob », qu'elle a l'impression d'être dans le travestissement. Interrogée par la Kommandantur, Cahun reprend les allures de Lucy Schwob qui la rendent méconnaissable :

Ils avaient enfin repéré le nom de «Schwob». Convoquée à la Kommandantur, j'y avais subi un interrogatoire d'allure bureaucratique. Je m'en étais tirée, pensais-je, à notre avantage. J'y étais allée - méconnaissable - en Lucy Schwob. Je vivais normalement, sous mon aspect Claude Cahun. Les bureaucrates avaient fait des excuses à la vieille dame en noir qui avait l'air si malade ${ }^{14}$.

La pratique artistique de Claude Cahun est avant tout une entreprise de résistance. Du même coup, une porosité s'établit chez cette artiste entre la lutte active, directement ancrée dans le réel (en témoignent les diverses ruses utilisées pendant la Résistance à Jersey) et la lutte plus spirituelle, qui la conduit à refuser tout carcan et toute catégorisation. La subversion des genres (sexuels) passant par la pratique du travestissement a établi une porosité entre la vie rêvée, artistique, et la vie réelle. L'attention portée au geste de nomination, qui pouvait avoir des conséquences extrêmement graves dans un contexte antisémite, est à la base du travail scriptural de Claude Cahun. Porter des masques est passé du statut de stratégie artistique à celui d'opération de survie. Dès lors, il est clair que le travestissement tel qu'il est utilisé par Cahun n'est pas simplement de l'ordre de la subversion humoristique. Il repose sur une véritable éthique qui consiste à penser que le danger vient d'une tendance à rabaisser l'humain à une étiquette, à des stéréotypes, tendance dont l'aboutissement avéré a pu être l'idéologie fasciste :

la modification ou suppression du nom propre m'est dictée par le sentiment profond du caractère sacré d'un être. Aucun nom, dès lors, n'est assez grand, n'est assez beau pour lui.

13. Claude Cahun, Le Muet dans la mêlée [1948], repris dans Écrits, op. cit., p. 627.

14. Ibid., p. 630. 
Pour peu qu'il me soit familier, sa multiplicité bien aimée, son ubiquité, sa faculté de me transformer l'univers, me contraignent, s'il me faut lié à lui le nommer malgré tout, à n'user que de termes élémentaires, de périphrases ou de charmes dérisoires, diminutifs de sons à peine audibles, impossibles à noter au moyen d'aucun alphabet, d'aucun signe préconçu ${ }^{15}$.

La subversion des genres littéraires qui participe d'une recherche toujours poussée plus loin de la justesse, c'est-à-dire de l'appréciation juste de la complexité des individus et du monde répond à un désir de se libérer des catégories, de se neutraliser, de façon à pouvoir lutter librement contre toute forme d'oppression.

\author{
Alexandra Bourse \\ Université Paris 13, Sorbonne Paris Cité, CENEL/PLÉIADE
}

15. Claude Cahun, « Confidences au miroir », op. cit., p. 586. 